\title{
On the Generalized Degenerate Tangent Numbers and Polynomials
}

\author{
Cheon Seoung Ryoo* \\ Department of Mathematics, Hannam University, Daejeon, Korea \\ *Corresponding author: ryoocs@hnu.kr
}

Received June 16, 2015; Revised August 26, 2015; Accepted September 19, 2015

\begin{abstract}
In [7], Ryoo introduced the generalized tangent numbers and polynomials. In this paper, our goal is to give generating functions of the degenerate generalized tangent numbers and polynomials. We also obtain some explicit formulas for degenerate generalized tangent numbers and polynomials.
\end{abstract}

Keywords: generalized tangent numbers and polynomials, degenerate generalized tangent numbers and polynomials

Cite This Article: Cheon Seoung Ryoo, "On the Generalized Degenerate Tangent Numbers and Polynomials." Turkish Journal of Analysis and Number Theory, vol. 3, no. 4 (2015): 104-107. doi: 10.12691/tjant-3-4-3.

\section{Introduction}

In [2], L. Carlitz introduced the degenerate Bernoulli polynomials. Recently, Feng Qi et al. [3] studied the partially degenerate Bernoull polynomials of the first kind in $p$-adic field. In [9], Ryoo constructed the degenerate tangent numbers and polynomials. In this paper, we introduce the degenerate generalized tangent numbers and polynomials. We also obtain some interesting properties for degenerate generalized tangent numbers and polynomials. Throughout this paper, we always make use of the following notations: $\mathbb{N}$ denotes the set of natural numbers and $\mathbb{Z}_{+}=\mathbb{N} \cup\{0\}, \mathbb{C}$ denotes the set of complex numbers. The tangent numbers are defined by means of the following generating function:

$$
\sum_{n=0}^{\infty} T_{n} \frac{t^{n}}{n !}=\frac{2}{e^{2 t}+1}\left(|t|<\frac{\pi}{2}\right) .
$$

Ryoo [6] defined tangent polynomials by multiplying $e^{x t}$ on the right side of the Eq. (1.1) as follows:

$$
\sum_{n=0}^{\infty} T_{n}(x) \frac{t^{n}}{n !}=\frac{2}{e^{2 t}+1} e^{x t} \quad\left(|t|<\frac{\pi}{2}\right) .
$$

Let $\chi$ be Dirichlet's character with conductor $d \in \mathbb{N}$ with $d=1(\bmod 2)$. Then the generalized tangent numbers associated with associated with $\chi, T_{n, \chi}$, are defined by the following generating function

$$
F_{\chi}(t)=\frac{2 \sum_{a=0}^{d-1} \chi(a)(-1)^{a} e^{2 a t}}{e^{2 d t}+1}=\sum_{n=0}^{\infty} T_{n, \chi} \frac{t^{n}}{n !} .
$$

We now consider the generalized tangent polynomials associated with $\chi, T_{n, \chi}(x)$, are also defined by

$$
\begin{aligned}
& F_{\chi}(x, t)=\left(\frac{2 \sum_{a=0}^{d-1} \chi(a)(-1)^{a} e^{2 a t}}{e^{2 d t}+1}\right) e^{x t} \\
& =\sum_{n=0}^{\infty} T_{n, \chi}(x) \frac{t^{n}}{n !} .
\end{aligned}
$$

When $\chi=\chi^{0}$, above (1.3) and (1.2) will become the corresponding definitions of the tangent numbers $T_{n}$ and polynomials $T_{n}(x)$ (see [6]). For more theoretical properties of the generalized tangent numbers and polynomials, the readers may refer to [7].

For a variable $t$, we consider the degenerate tangent polynomials which are given by the generating function to be

$$
\frac{2}{(1+\lambda t)^{2 / \lambda}+1}(1+\lambda t)^{x / \lambda}=\sum_{n=0}^{\infty} T_{n, \lambda}(x) \frac{t^{n}}{n !} .
$$

When $x=0, T_{n, \lambda}(0)=T_{n, \lambda}$ are called the degenerate tangent numbers(see [8]).

We recall that the classical Stirling numbers of the first kind $S_{1}(n, k)$ and $S_{2}(n, k)$ are defined by the relations (see $[9,10])$

$$
(x)_{n}=\sum_{k=0}^{n} S_{1}(n, k) x^{k} \text { and } x^{n}=\sum_{k=0}^{n} S_{2}(n, k)(x)_{k},
$$

respectively. Here $(x)_{n}=x(x-1) \cdots(x-n+1)$ denotes the falling factorial polynomial of order $n$. The numbers $S_{2}(n, m)$ also admit a representation in terms of a generating function

$$
\sum_{n=m}^{\infty} S_{2}(n, m) \frac{t^{n}}{n !}=\frac{\left(e^{t}-1\right)^{m}}{m !}
$$


We also have

$$
\sum_{n=m}^{\infty} S_{1}(n, m) \frac{t^{n}}{n !}=\frac{(\log (1+t))^{m}}{m !} .
$$

The generalized falling factorial $(x \mid \lambda)_{n}$ with increment $\lambda$ is defined by

$$
(x \mid \lambda)_{n}=\prod_{k=0}^{n-1}(x-\lambda k)
$$

for positive integer $n$, with the convention $(x \mid \lambda)_{0}=1$. We also need the binomial theorem:

for a variable $x$,

$$
(1+\lambda t)^{x / \lambda}=\sum_{n=0}^{\infty}(x \mid \lambda)_{n} \frac{t^{n}}{n !}
$$

\section{On the Degenerate Generalized \\ Tangent Polynomials}

In this section, we define the degenerate generalized tangent numbers and polynomials, and we obtain explicit formulas for them. Let $\chi$ be Dirichlet's character with conductor $d \in \mathbb{N}$ with $d \equiv 1(\bmod 2)$. Then the degenerate generalized tangent numbers associated with $\chi, T_{n, \chi}$, are defined by the following generating function

$$
\begin{aligned}
& \frac{2 \sum_{a=0}^{d-1}(-1)^{a} \chi(a)(1+\lambda t)^{2 a / \lambda}}{(1+\lambda t)^{2 d / \lambda}+1}(1+\lambda t)^{x / \lambda} \\
& =\sum_{n=0}^{\infty} T_{n, \chi, \lambda}(x) \frac{t^{n}}{n !} .
\end{aligned}
$$

When $\quad x=0, \quad T_{n, \chi, \lambda}(0)=T_{n, \chi, \lambda}$ are called the generalized degenerate tangent numbers.

From (2.1) and (1.4), we note that

$$
\begin{aligned}
& \sum_{n=0}^{\infty} \lim _{\lambda \rightarrow 0} T_{n, \chi, \lambda}(x) \frac{t^{n}}{n !} \\
& =\lim _{\lambda \rightarrow 0} \frac{2 \sum_{a=0}^{d-1}(-1)^{a} \chi(a)(1+\lambda t)^{2 a / \lambda}}{(1+\lambda t)^{2 d / \lambda}+1}(1+\lambda t)^{x / \lambda} \\
& =\left(\frac{2 \sum_{a=0}^{d-1} \chi(a)(-1)^{a} e^{2 a t}}{e^{2 d t}+1}\right) e^{x t} \\
& =\sum_{n=0}^{\infty} T_{n, \chi}(x) \frac{t^{n}}{n !} .
\end{aligned}
$$

Thus, we get

$$
\lim _{\lambda \rightarrow 0} T_{n, \chi, \lambda}(x)=T_{n, \chi}(x),(n \geq 0) .
$$

From (2.1) and (1.5), we have

$$
\begin{aligned}
& \sum_{n=0}^{\infty} T_{n, \chi, \lambda}(x) \frac{t^{n}}{n !} \\
& =\frac{2 \sum_{a=0}^{d-1}(-1)^{a} \chi(a)(1+\lambda t)^{2 a / \lambda}}{(1+\lambda t)^{2 d / \lambda}+1}(1+\lambda t)^{x / \lambda} \\
& =\left(\sum_{m=0}^{\infty} T_{m, \chi, \lambda} \frac{t^{m}}{m !}\right)\left(\sum_{t=0}^{\infty}(x \mid \lambda)_{l} \frac{t^{l}}{l !}\right) \\
& =\sum_{n=0}^{\infty}\left(\sum_{l=0}^{n}\left(\begin{array}{l}
n \\
l
\end{array}\right) T_{l, \lambda}(x \mid \lambda)_{n-l}\right) \frac{t^{n}}{n !} .
\end{aligned}
$$

Therefore, by (2.1) and (2.2), we obtain the following theorem.

Theorem 1. For $n \geq 0$, we have

$$
T_{n, \chi, \lambda}(x)=\sum_{l=0}^{n}\left(\begin{array}{l}
n \\
l
\end{array}\right) T_{l, \chi, \lambda}(x \mid \lambda)_{n-l} .
$$

For $d \in \mathbb{N}$ with $d \equiv 1(\bmod 2)$, we have

$$
\begin{aligned}
& \sum_{n=0}^{\infty} T_{n, \chi, \lambda}(x) \frac{t^{n}}{n !} \\
& =\frac{2 \sum_{a=0}^{d-1}(-1)^{a} \chi(a)(1+\lambda t)^{2 a / \lambda}}{(1+\lambda t)^{2 d / \lambda}+1}(1+\lambda t)^{x / \lambda} \\
& =\frac{2}{(1+\lambda t)^{2 d / \lambda}+1}(1+\lambda t)^{x / \lambda} \sum_{l=0}^{d-1}(-1)^{l} \chi(l)(1+\lambda t)^{2 l / \lambda} \\
& =\sum_{n=0}^{\infty}\left(d^{n} \sum_{l=0}^{d-1}(-1)^{l} \chi(l) T_{n, \lambda / d}\left(\frac{2 l+x}{d}\right)\right) \frac{t^{n}}{n !} .
\end{aligned}
$$

By comparing coefficients of $\frac{t^{m}}{m !}$ in the above equation, we have the following theorem.

Theorem 2. Let $\chi$ be Dirichlet's character with conductor $d \in \mathbb{N}$ with $d \equiv 1(\bmod 2)$. Then we have

(1) $T_{m, \chi, \lambda}(x)=d^{m} \sum_{a=0}^{d-1} \chi(a)(-1)^{a} T_{m, \lambda / d}\left(\frac{2 a+x}{d}\right)$,

(2) $T_{m, \chi, \lambda}=d^{m} \sum_{a=0}^{d-1} \chi(a)(-1)^{a} T_{m, \lambda / d}\left(\frac{2 a}{d}\right)$.

From (2.1), we can derive the following relation:

$$
\begin{aligned}
& \sum_{m=0}^{\infty}\left(T_{m, \chi, \lambda}(2 d)+T_{m, \chi, \lambda}(x)\right) \frac{t^{m}}{m !} \\
= & \frac{2 \sum_{a=0}^{d-1}(-1)^{a} \chi(a)(1+\lambda t)^{2 a / \lambda}}{(1+\lambda t)^{2 d / \lambda}+1}(1+\lambda t)^{x / \lambda} \\
+ & \frac{2 \sum_{a=0}^{d-1}(-1)^{a} \chi(a)(1+\lambda t)^{2 a / \lambda}}{(1+\lambda t)^{2 d / \lambda}+1} \\
= & 2 \sum_{a=0}^{d-1}(-1)^{a} \chi(a)(1+\lambda t)^{2 a / \lambda} \\
= & \sum_{m=0}^{\infty}\left(2 \sum_{a=0}^{d-1}(-1)^{a} \chi(a)(2 a \mid \lambda)_{m}\right) \frac{t^{m}}{m !} .
\end{aligned}
$$


By comparing of the coefficients $\frac{t^{m}}{m !}$ on the both sides of (2.4), we have the following theorem.

Theorem 3. For $n \in \mathbb{Z}_{+}$, we have

$$
T_{m, \chi, \lambda}(2 d)+T_{m, \chi, \lambda}=2 \sum_{a=0}^{d-1}(-1)^{a} \chi(a)(2 a / \lambda)_{m} .
$$

By (2.1), we have

$$
\begin{aligned}
& \sum_{m=0}^{\infty}\left(T_{m, \chi, \lambda}(x+2 d)+T_{m, \chi, \lambda}(x)\right) \frac{t^{m}}{m !} \\
= & 2 \sum_{a=0}^{d-1}(-1)^{a} \chi(a)(1+\lambda t)^{2 a / \lambda}(1+\lambda t)^{x / \lambda} \\
= & \sum_{m=0}^{\infty}\left(2 \sum_{a=0}^{d-1}(-1)^{a} \chi(a) \sum_{l=0}^{m}\left(\begin{array}{l}
m \\
l
\end{array}\right)(x \mid \lambda)_{l}(2 a \mid \lambda)_{m-l}\right) \frac{t^{m}}{m !} .
\end{aligned}
$$

By comparing of the coefficients $\frac{t^{m}}{m !}$ on the both sides of (2.5), we have the following theorem.

Theorem 4. For $n \in \mathbb{Z}_{+}$, we have

$$
\begin{aligned}
& T_{m, \chi, \lambda}(x+2 d)+T_{m, \chi, \lambda}(x) \\
& =2 \sum_{a=0}^{d-1}(-1)^{a} \chi(a) \sum_{l=0}^{m}\left(\begin{array}{l}
m \\
l
\end{array}\right)(x \mid \lambda)_{l}(2 a \mid \lambda)_{m-l} .
\end{aligned}
$$

From (2.1), we have

$$
\begin{aligned}
& \sum_{n=0}^{\infty} T_{n, \chi, \lambda}(x+y) \frac{t^{n}}{n !} \\
= & \frac{2 \sum_{a=0}^{d-1}(-1)^{a} \chi(a)(1+\lambda t)^{2 a / \lambda}}{(1+\lambda t)^{2 d / \lambda}+1}(1+\lambda t)^{(x+y) / \lambda} \\
= & \frac{2 \sum_{a=0}^{d-1}(-1)^{a} \chi(a)(1+\lambda t)^{2 a / \lambda}}{(1+\lambda t)^{2 d / \lambda}+1} \\
& \times(1+\lambda t)^{x / \lambda}(1+\lambda t)^{y / \lambda} \\
= & \left(\sum_{n=0}^{\infty} T_{n, \chi, \lambda}(x) \frac{t^{n}}{n !}\right)\left(\sum_{n=0}^{\infty}(y \mid \lambda)_{n} \frac{t^{n}}{n !}\right) \\
= & \sum_{n=0}^{\infty}\left(\sum_{l=0}^{n}\left(\begin{array}{l}
n \\
l
\end{array}\right) T_{l, \chi, \lambda}(y \mid \lambda)_{n-l}\right) \frac{t^{n}}{n !} .
\end{aligned}
$$

Therefore, by (2.6), we have the following theorem.

Theorem 5. For $n \in \mathbb{Z}_{+}$, we have

$$
T_{n, \chi, \lambda}(x+y)=\sum_{k=0}^{n}\left(\begin{array}{l}
n \\
k
\end{array}\right) T_{k, \chi, \lambda}(x)(y \mid \lambda)_{n-k} .
$$

From Theorem 5, we note that $T_{n, \chi, \lambda}(x)$ is a Sheffer sequence.

By replacing $t$ by $\frac{e^{\lambda t}-1}{\lambda}$ in (2.1), we obtain

$$
\begin{aligned}
& \frac{2 \sum_{a=0}^{d-1} \chi(a)(-1)^{a} e^{2 a t}}{e^{2 d t}+1} e^{x t} \\
= & \sum_{n=0}^{\infty} T_{n, \chi, \lambda}(x)\left(\frac{e^{\lambda t}-1}{\lambda}\right)^{n} \frac{1}{n !} \\
= & \sum_{n=0}^{\infty} T_{n, \chi, \lambda}(x) \lambda^{-n} \sum_{m=n}^{\infty} S_{2}(m, n) \lambda^{m} \frac{t^{m}}{m !} \\
= & \sum_{m=0}^{\infty}\left(\sum_{n=0}^{m} T_{n, \chi, \lambda}(x) \lambda^{m-n} S_{2}(m, n) \frac{t^{m}}{m !} .\right.
\end{aligned}
$$

Thus, by (2.7) and (1.4), we have the following theorem. Theorem 6. For $n \in \mathbb{Z}_{+}$, we have

$$
T_{m, \chi}(x)=\sum_{n=0}^{m} \lambda^{m-n} T_{n, \chi, \lambda}(x) S_{2}(m, n) .
$$

By replacing $t$ by $\log (1+\lambda t)^{1 / \lambda}$ in (1.4), we have

$$
\begin{aligned}
& \sum_{n=0}^{\infty} T_{n, \chi}\left(\log (1+\lambda t)^{1 / \lambda}\right)^{n} \frac{1}{n !} \\
& =\frac{2 \sum_{a=0}^{d-1}(-1)^{a} \chi(a)(1+\lambda t)^{2 a / \lambda}}{(1+\lambda t)^{2 d / \lambda}+1}(1+\lambda t)^{x / \lambda} \\
& =\sum_{m=0}^{\infty} T_{m, \chi, \lambda}(x) \frac{t^{m}}{m !}
\end{aligned}
$$

and

$$
\begin{aligned}
& \sum_{n=0}^{\infty} T_{n, \chi}\left(\log (1+\lambda t)^{1 / \lambda}\right)^{n} \frac{1}{n !} \\
& =\sum_{m=0}^{\infty}\left(\sum_{n=0}^{\infty} T_{n, \chi}(x) \lambda^{m-n} S_{1}(m, n)\right) \frac{t^{m}}{m !} .
\end{aligned}
$$

Thus, by (2.8) and (2.9), we have the following theorem.

Theorem 7. For $n \in \mathbb{Z}_{+}$, we have

$$
T_{m, \chi, \lambda}(x)=\sum_{n=0}^{\infty} \lambda^{m-n} T_{n, \chi}(x) S_{1}(m, n) .
$$

\section{References}

[1] L. Carlitz, Degenerate Stirling, Bernoulli and Eulerian numbers, Utilitas Math. 15(1979), 51-88.

[2] L. Carlitz, A degenerate Staudt-Clausen theorem, Arch. Math. (Basel) 7(1956), 28-33.

[3] F. Qi, D. V. Dolgy, T. Kim, C. S. Ryoo, On the partially degenerate Bernoulli polynomials of the first kind, Global Journal of Pure and Applied Mathematics, 11(2015), 2407-2412.

[4] T. Kim, Barnes' type multiple degenerate Bernoulli and Euler polynomials, Appl. Math. Comput. 258(2015), 556-564.

[5] H. Ozden, I. N. Cangul, Y. Simsek, Remarks on $q$-Bernoulli numbers associated with Daehee numbers, Adv. Stud. Contemp. Math. 18(2009), no. 1, 41-48.

[6] C. S. Ryoo, A Note on the tangent numbers and polynomials, Adv. Studies Theor. Phys., 7(2013), no. 9, 447-454.

[7] C. S. Ryoo, Generalized tangent numbers and polynomials associated with $p$-adic integral on $\mathbb{Z} p$, Applied Mathematical Sciences, 7(2013), no. 99, 4929-4934. 
[8] C. S. Ryoo, Some identities on the $(h ; q)$-tangent polynomials and Bernstein Polynomials, Applied Mathematical Sciences, 8(2014), no. 75, 3747-3753.

[9] C. S. Ryoo, Notes on degenerate tangent polynomials, to appear in Global Journal of Pure and Applied Mathematics, Volume 11, number 5(2015), pp. 3631-3637.
[10] P. T. Young, Degenerate Bernoulli polynomials, generalized factorial sums, and their applications, Journal of Number Theorey, 128(2008), 738-758. 